University of New Orleans

ScholarWorks@UNO

$11-1978$

\title{
Consequences of light reflection at the interface between two transparent media such that the angle of refraction is $45^{\circ}$
}

R. M.A. Azzam

University of New Orleans, razzam@uno.edu

Follow this and additional works at: https://scholarworks.uno.edu/ee_facpubs

Part of the Electrical and Electronics Commons, and the Physics Commons

\section{Recommended Citation}

R. M. A. Azzam, "Consequences of light reflection at the interface between two transparent media such that the angle of refraction is $45^{\circ}$, " J. Opt. Soc. Am. 68, 1613-1615 (1978)

This Article is brought to you for free and open access by the Department of Electrical Engineering at ScholarWorks@UNO. It has been accepted for inclusion in Electrical Engineering Faculty Publications by an authorized administrator of ScholarWorks@UNO. For more information, please contact scholarworks@uno.edu. 
${ }^{6}$ J. C. Owens, Appl. Opt. 6, 51 (1967).

${ }^{7} \mathrm{~F}$. Spieweck, in Proceedings of the LASER-77 Opto-Electronics Conference, edited by W. Waidelich, (Science and Technology Press Limited, Guildford, England, 1977), p. 130; F. Spieweck, Metrologia, 12, 43 (1976).
${ }^{8}$ C. Wieman and T. W. Hänsch, Phys. Rev. Lett. 36, 1170 (1976).

${ }^{9}$ A. Javan, J. Opt. Soc. Am., 67, 1413 (1977).

${ }^{10}$ W. R. C. Rowley, K. C. Shotton, and P. T. Woods, in Laser Spectroscopy III, edited by J. L. Hall and J. L. Carlsten, (SpringerVerlag, New York, 1977), p. 425.

\title{
Consequences of light reflection at the interface between two transparent media such that the angle of refraction is $45^{\circ}$
}

\author{
R. M. A. Azzam \\ Hematology Division and Department of Internal Medicine, The University of Nebraska Medical Center, Omaha, Nebraska 68105
}

(Received 22 May 1978)

\begin{abstract}
The reflection of light at the interface between two transparent, homogeneous and optically isotropic media such that the angle of refraction is $45^{\circ}$ has the following interesting consequences: (i) the interface relfectivity for the parallel $(p)$ polarization is the square of that for the perpendicular $(s)$ polarization; (ii) the angular deviation of the propagation direction upon refraction is equal to the polarization (or ellipsometric) angle $\psi$; and (iii) the complex reflection coefficient for the $p$ polarization is stationary, in both magnitude and phase, with respect to small charges of the (refractive and/or absorptive) optical properties of either one or both media. Furthermore, it follows from (iii) that: (iv) reflectivity of the $p$ polarization, considered as a function of the ratio of refractive indices of the two media, is at a maximum; and (v) changes of the absolute magnitude and absolute phase of the reflection coefficient for the $s$ polarization, caused by small perturbations of the optical properties, can be measured directly by ellipsometry.
\end{abstract}

When considering the reflection of light, or any other electromagnetic radiation, at the interface between two transparent, homogeneous, and isotropic media, certain angles of incidence are distinguished for their special significance. The most well known are the Brewster angle and the critical angle. At the Brewster angle light polarized parallel to the plane of incidence is completely extinguished upon reflection, and the associated reflection phase shift experiences a jump of $\pi$ as the angle of incidence is swept past the Brewster angle. The critical angle marks the onset of total internal reflection and exists only when light is incident on the interface from the medium of higher refractive index. To these special angles we may add $0^{\circ}, 45^{\circ}$, and $90^{\circ}$ which correspond to normal incidence, symmetrical incidence, and grazing incidence, respectively.

Incidence at $45^{\circ}$ has the little known distinction of making the interface reflectivity for the $p$ polarization equal to the square of that for the $s$ polarization. ${ }^{1,2}$ We have known ${ }^{3}$ that incidence at $45^{\circ}$ has other interesting consequences (see the following).

In this Letter we introduce another angle of incidence of special significance, namely the angle of incidence that makes the angle of refraction equal to $45^{\circ}$. At this angle we will prove that (i) the reflectivity for the $p$ polarization is the square of the reflectivity for the $s$ polarization; and (ii) the angular deviation of the propagation direction upon refraction is equal to the polarization or ellipsometric angle $\psi$. 'These two properties also apply when the angle of incidence is $45^{\circ} .3$ In addition, at this new angle, the complex Fresnel reflection coefficient for the $p$ polarization is stationary, in both magnitude and phase, with respect to small changes of the optical properties of either one or both media. Because no similar effect is encountered with the $s$ polarization, small changes of the complex reflection coefficient of $s$ polarized light can be measured directly by ellipsometry. 4

Consider the reflection of light at an angle of incidence $\phi$ by the interface between two homogeneous and isotropic media with refractive indices $n$ and $N$. The angle of refraction $\phi^{\prime}$ is determined by Snell's law:

$$
n \sin \phi=N \sin \phi^{\prime} .
$$

The Fresnel reflection coefficients for the $p$ and $s$ polarizations (with linear electric field vibrations parallel and perpendicular to the plane of incidence, respectively) can be expressed in terms of $\phi$ and $\phi^{\prime}$ only as ${ }^{5}$

$$
\begin{aligned}
& r_{p}=\tan \left(\phi-\phi^{\prime}\right) / \tan \left(\phi+\phi^{\prime}\right), \\
& r_{s}=-\sin \left(\phi-\phi^{\prime}\right) / \sin \left(\phi+\phi^{\prime}\right) .
\end{aligned}
$$

Alternatively, $r_{p}$ and $r_{s}$ can be written as functions of $\phi$ and the refractive index ratio

$$
\nu=N / n,
$$

by eliminating $\phi^{\prime}$ from Eqs. (2) and (3) using Eq. (1):

$$
\begin{gathered}
r_{p}=\frac{\nu^{2} \cos \phi-\left(\nu^{2}-\sin ^{2} \phi\right)^{1 / 2}}{\nu^{2} \cos \phi+\left(\nu^{2}-\sin ^{2} \phi\right)^{1 / 2}} \\
r_{s}=\frac{\cos \phi-\left(\nu^{2}-\sin ^{2} \phi\right)^{1 / 2}}{\cos \phi+\left(\nu^{2}-\sin ^{2} \phi\right)^{1 / 2}}
\end{gathered}
$$

From Eq. (1), the angle of incidence $\phi$ that makes the angle of refraction $\phi^{\prime}=45^{\circ}$ is given by

$$
\sin \phi=\nu / \sqrt{2} .
$$

This angle exists always in internal reflection $(\nu<1)$, and exists in external reflection only if $\nu<\sqrt{2}$. Table I gives this angle for two common interfaces. 
TABLE I. Some values of the angle of incidence $\phi$ for light refraction at $45^{\circ} .^{*}$

\begin{tabular}{ccccc}
\hline \hline Interface & air-water & water-air & air-glass & glass-air \\
\hline$\nu$ & 1.33 & $1 / 1.33$ & 1.5 & $1 / 1.5$ \\
$\nu \stackrel{\sqrt{2} ?}{2}=\sqrt{2}$ & $<\sqrt{2}$ & $>\sqrt{2}$ & $<\sqrt{2}$ \\
$\phi$ (degrees) & 70.127 & 32.118 & nonexistent & 28.126 \\
\hline \hline
\end{tabular}

* We assume that the wavelength of light is in the visible spectrum.

When the angle of refraction $\phi^{\prime}=45^{\circ}$, Eqs. (2) and (3) become

$$
\begin{gathered}
r_{p}=-\tan ^{2}\left(\phi-45^{\circ}\right), \\
r_{s}=-\tan \left(\phi-45^{\circ}\right),
\end{gathered}
$$

as can be proved by the application of some trigonometry. ${ }^{6}$ From Eqs. (8) and (9) it is evident that

$$
r_{p}=-r_{s}^{2}
$$

and

$$
R_{p}=R_{s}^{2}
$$

so that the reflectance $\left(\mathrm{R}=|r|^{2}\right)$ for the $p$ polarization is the square of the reflectance for the $s$ polarization. Equation (11) is satisfied also when the angle of incidence $\phi=45^{\circ} .1-3$ Equations (8) and (9) show that, when the angle of refraction is $45^{\circ}$, the reflection phase shift for the $p$ polarization is $\pi$, while that for the $s$ polarization is either $\pi$ (external incidence, $\phi>45^{\circ}$ ) or 0 (internal incidence $\phi<45^{\circ}$ ).

The ratio of $r_{p}$ and $r_{s}$ is given by

$$
\begin{aligned}
\rho & =r_{p} / r_{s} \\
& =\tan \nu e^{j \Delta} \\
& =\tan \left(\phi-45^{\circ}\right),
\end{aligned}
$$

as can be seen from Eqs. (7) and (8), and

$$
\psi=\left|\phi-45^{\circ}\right| \text {. }
$$

Equation (13) tells us that the angular beam deviation upon refraction when $\phi^{\prime}=45^{\circ}$ is equal to the polarization angle $\psi$, Fig. 1. We have found that a similar relation applies when the angle of incidence is $45^{\circ} .3$ This relation between propagation directions and polarization directions is probably one of the simplest possible links between geometrical optics and physical optics.

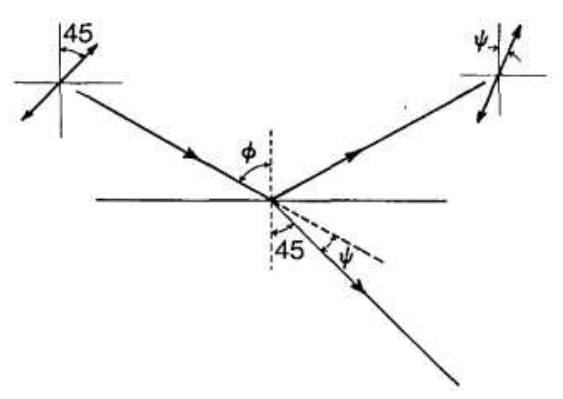

FIG. 1. When light is incident on the interface between two transparent media such that the angle of refraction is $45^{\circ}$, the angulardeviation of the propagation direction caused by refraction equals the polarization (ellipsometric) angle $\psi$.

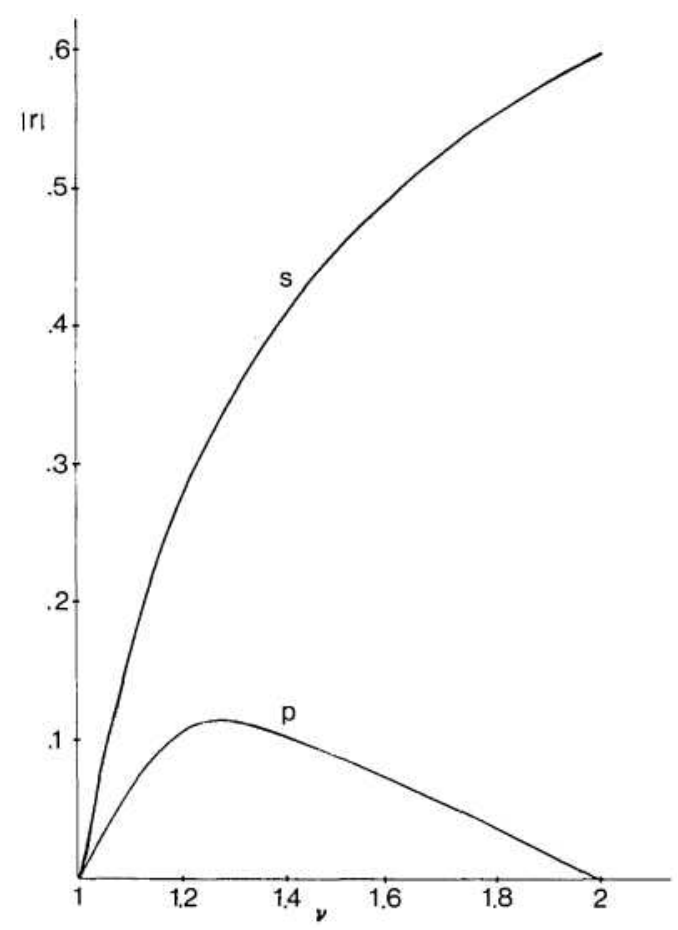

FIG. 2. The magnitude $|r|$ of Fresnel's reflection coefficients for the parallel $(p)$ and perpendicular $(s)$ polarizations as functions of the refractive index ratio $\nu$ between $\nu=1$ and $\nu=2$. We assume light reflection at the interface between two transparent media at the fixed angle of incidence $\phi=\tan ^{-1} 2=63.435^{\circ}$. Note that $\left|r_{p}\right|$ goes through a peak when $\nu=\sqrt{2}$ $\sin \phi=1.265$ (i.e. when the light is refracted at $45^{\circ}$ ), whereas $\left|r_{s}\right|$ increases monotonically. $r_{p}$ and $r_{s}$ are both real and negative.

When the angle of incidence is fixed, and the refractive index ratio $\nu$ is varied, the reflection coefficient for the $p$ polarization as a function of $\nu, r_{p}(\nu)$, must go through a peak between $\nu=1$ and $\nu=\tan \phi$, because ${ }^{7} r_{p}(1)=r_{p}(\tan \phi)=0$. To determine the position of the peak (i.e., the value of $\nu$ at which it occurs), we find the derivative of $r_{p}$ with respect to $\nu$ and equate it to zero. From Eq. (5) we have

$$
\frac{\partial r_{p}}{\partial \nu}=\frac{2 \nu \cos \phi\left(\nu^{2}-2 \sin ^{2} \phi\right)}{\left(\nu^{2}-\sin ^{2} \phi\right)^{1 / 2}\left[\nu^{2} \cos \phi+\left(\nu^{2}-\sin ^{2} \phi\right)^{1 / 2}\right]^{2}},
$$

which is equal to zero when $\nu=\sqrt{2} \sin \phi$, i.e., when the angle of refraction is $45^{\circ}$ [see Eq. (7)]. The corresponding derivative of $r_{s}$ with respect to $\nu$,

$$
\frac{\partial r_{s}}{\partial \nu}=\frac{-2 \nu \cos \phi}{\left(\nu^{2}-\sin ^{2} \phi\right)^{1 / 2}\left[\cos \phi+\left(\nu^{2}-\sin ^{2} \phi\right)^{1 / 2}\right]^{2}},
$$

does not vanish for any value of $\nu$ in the range ${ }^{8} 0<\nu<\infty$.

As an illustration, Fig. 2 shows $\left|r_{p}\right|$ and $\left|r_{s}\right|$ vs $\nu$ between $\nu=1$ and $\nu=2$, when the angle of incidence is fixed at $\phi=$ $\tan ^{-1} 2=63.435^{\circ}$. Notice that $\left|r_{p}\right|$ goes through a peak at $\nu$ $=\sqrt{2} \sin 63.435^{\circ}=1.265$, as expected, while $\left|r_{s}\right|$ increases monotonically.

The vanishing of $\partial r_{p} / \partial \nu$ when the angle of refraction is $45^{\circ}$ means that $r_{p}$ is stationary in both magnitude and phase with respect to small variations of the optical properties of one or both media. This is significant in modulation spectroscopy ${ }^{9}$ where small changes of the optical properties are induced by a modulating field such as temperature, stress, electric or magnetic field. We have found above that modulation of the 
optical properties does not show up in reflection under the following conditions: (i) the media are transparent, (ii) the incident light is $p$ polarized, and (iii) the angle of refraction is $45^{\circ}$.

The reflection coefficient for the $s$ polarization does change when $\nu$ is varied in the neighborhood of $\nu=\sqrt{2} \sin \phi$, because $\partial r_{s} / \partial \nu \neq 0$. The changes of the absolute magnitude and phase of $r_{s}$ can be measured directly by ellipsometry. In this case small changes of $r_{s}$ show directly as small changes of the ellipsometric ratio of reflection coefficients $\rho=r_{p} / r_{s}$. Because $r_{p}$ is stationary, we have

$$
\frac{\delta \rho}{\rho}=-\frac{\delta r_{s}}{r_{s}}=-\frac{1}{r_{s}} \frac{\partial r_{s}}{\partial \nu} \delta \nu .
$$

If we substitute $\nu=\sqrt{2} \sin \phi$ in Eqs. (6) and (15), we find that

$$
-\frac{1}{r_{s}} \frac{\partial r_{s}}{\partial \nu}=\frac{2 \sqrt{2} \cos \phi}{\cos 2 \phi}
$$

or

$$
-\frac{1}{r_{s}} \frac{\partial r_{s}}{\partial \nu}=2 \sqrt{2}\left(1-1 / 2^{2}\right)^{1 / 2} /\left(1-\nu^{2}\right) .
$$

If we take the differential of $\rho=\tan \psi e^{\mathrm{j} \Delta}$, we get

$$
\delta \rho, \rho=2 \delta \psi / \sin 2 \psi+j \delta \Delta .
$$

In general, $\delta \nu$ can be written as

$$
\delta \nu=\delta n-j \delta k,
$$

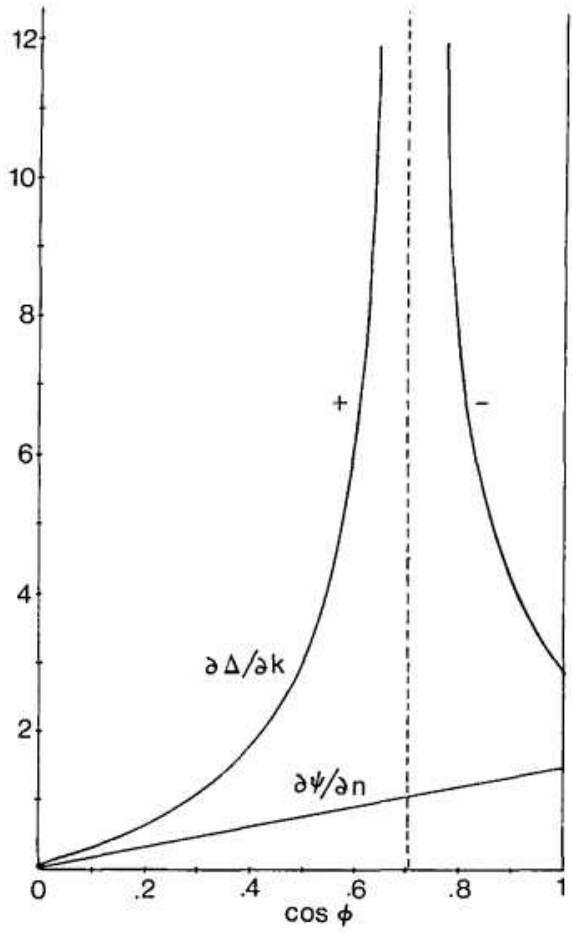

FIG. 3. Ellipsometric partial derivatives $\partial \Delta / \partial k$ and $\partial \psi / \partial n$ vs $\cos \phi$, where $\phi$ is the angle of incidence that makes the angle of refraction equal to $45^{\circ}$. The + and - signs indicate positive and negative values of $\partial \Delta / \partial k$. where $\delta n$ and $\delta k$ are the changes of the relative refractive index and relative extinction coefficient, respectively. If we substitute Eqs. (17), (19), and (20) into Eq. (16), and use Eq. (13), we obtain

$$
\begin{gathered}
\delta \psi=(\sqrt{2} \cos \phi) \delta n, \\
\delta \Delta=(-2 \sqrt{2} \cos \phi / \cos 2 \phi) \delta k,
\end{gathered}
$$

and conversely,

$$
\begin{gathered}
\delta n=(1 / \sqrt{2} \cos \phi) \delta \psi, \\
\delta k=(-\cos 2 \phi / 2 \sqrt{2} \cos \phi) \delta \Delta .
\end{gathered}
$$

Equations (21) give the changes of the ellipsometric (polarization) parameters $\delta \psi$ and $\delta \Delta$ that are caused by a small change $\delta \nu=\delta n-j \delta k$ of the complex refractive index ratio $\nu$. Notice that $\delta n$ and $\delta k$ cause $\delta \psi$ and $\delta \Delta$, respectively. Equations (22) show how perturbations $\delta \nu=\delta n-j \delta k$ of the complex refractive index ratio $\nu$ can be easily determined from measurable changes $\delta \psi$ and $\delta \Delta$ of the ellipsometric parameters. Figure 3 shows

$$
\frac{\partial \psi}{\partial n}=\frac{\delta \psi}{\delta n}=\sqrt{2} \cos \phi
$$

and

$$
\frac{\partial \Delta}{\partial k}=\frac{\delta \Delta}{\delta k}=\frac{-2 \sqrt{2} \cos \phi}{\cos 2 \phi}
$$

as functions of $\cos \phi$. Notice that $|\partial \Delta / \partial k| \rightarrow \infty$ as $\phi \rightarrow 45^{\circ}$, which occurs as $\nu \rightarrow 1$, i.e., when the difference between the optical properties of the two media approach zero. ${ }^{10}$ For example, when $\cos \phi=0.7$ (or $\left.\phi=45.57^{\circ}\right), \partial \Delta / \partial k=98.995$ radians per unit of $k$. This means that an induced change of $k$ as small as $1 \times 10^{-6}$ causes a change of $\Delta$ equal to $0.006^{\circ}$, which is measurable by present-day ellipsometers. It should be observed, however, that as $\nu \rightarrow 1$ (and $\phi \rightarrow 45^{\circ}$ ), the intensity of the reflected beam $\rightarrow 0$, so that an intense light source has to be used to utilize the large values of $\partial \Delta / \partial k$.

IS. P. F. Humphreys-Owen, "Comparison of reflection methods for measuring optical constants without polarimetric analysis, and proposal for new methods based on the Brewster angle," Proc. Roy. Soc. Lond. A 77, 949 957 (1961).

${ }^{2} \mathrm{D}$. W. Berreman, "Simple relation between reflectances of polarized components of a beam when the angle of incidence is 45 degrees," J. Opt. Soc. Am. 56, 1784 (1966).

${ }^{3}$ R. M. A. Azzam, "On the reflection of light at $45^{\circ}$ angle of incidence," Opt. Acta (in press).

${ }^{4}$ For refraction at $45^{\circ}$ we will show that the Fresnel coefficients are interrelated by $r_{p}=-r_{s}^{2}$, so that ellipsometry measures $r_{p} / r_{s}=-r_{s}$. For incidence at $45^{\circ}$, we have $r_{p}=r_{s}^{2}$ and $r_{p} / r_{s}=r_{s}$ (Ref. 3).

${ }^{5}$ We assume the Nebraska (Muller) conventions. See R. H. Muller, "Definitions and conventions in ellipsometry," Surf. Sci. 16, 14-33 (1969).

${ }^{6}$ The proof is analogous to one to be found in Ref. 3 .

$\tau_{\nu}=\tan \phi$ is Brewster's condition for $r_{p}=0$.

$8 \partial r_{p} / \partial \nu$ and $\partial r_{s} / \partial \nu$ both vanish in the limiting cases of $\nu=0, \infty$.

${ }^{9}$ See, for example, Vol. 37 of Surface Science: Proceedings of the First International Conference on Modulation Spectroscopy, Edited by B. O. Seraphin, (North-Holland, Amsterdam, 1973).

${ }^{10}$ This corresponds, for example, to interfaces between the liquid and solid phases of the same substance. 